University of Rhode Island

DigitalCommons@URI

Nonequilibrium Statistical Physics

Physics Open Educational Resources

10-19-2015

\title{
09. Linear Response and Equilibrium Dynamics
}

Gerhard Müller

University of Rhode Island, gmuller@uri.edu

Follow this and additional works at: https://digitalcommons.uri.edu/nonequilibrium_statistical_physics

Part of the Physics Commons

\section{Abstract}

Part nine of course materials for Nonequilibrium Statistical Physics (Physics 626), taught by Gerhard Müller at the University of Rhode Island. Entries listed in the table of contents, but not shown in the document, exist only in handwritten form. Documents will be updated periodically as more entries become presentable.

Updated with version 2 on 5/3/2016.

\section{Recommended Citation}

Müller, Gerhard, "09. Linear Response and Equilibrium Dynamics" (2015). Nonequilibrium Statistical Physics. Paper 9.

https://digitalcommons.uri.edu/nonequilibrium_statistical_physics/9

This Course Material is brought to you for free and open access by the Physics Open Educational Resources at DigitalCommons@URI. It has been accepted for inclusion in Nonequilibrium Statistical Physics by an authorized administrator of DigitalCommons@URI. For more information, please contact digitalcommons-group@uri.edu. 


\section{Contents of this Document ${ }_{[\text {[ntes] }}$}

9. Linear Response and Equilibrium Dynamics

- Overview [nln24]

- Many-body system perturbed by radiation field [nln25]

- Response function and generalized susceptibility [nln26]

- Kubo formula for response function [nln27]

- Symmetry properties [nln30]

- Kramers-Kronig dispersion relations [nln37]

- Causality property of response function [nex63]

- Energy transfer between system and radiation field [nln38]

- Reactive and absorptive part of response function [nex64]

- Fluctuation-dissipation theorem (quantum and classical) [nln39]

- Moment expansion [nln78]

- Spectral representations [nex65]

- Linear response of classical relaxator [nex66]

- Dielectric relaxation in liquid water [nln76]

- Linear response of classical oscillator [nex67]

- Scattering process and dynamic structure factor [nln89]

- Scattering from free atoms [nln93]

- Scattering from atoms bound to lattice [nln94]

- Scattering from a harmonic crystal [nln95]

- Magnetic resonance or scattering [nln97] 


\section{Linear response and equilibrium dynamics ${ }_{[\ln 24]}$}

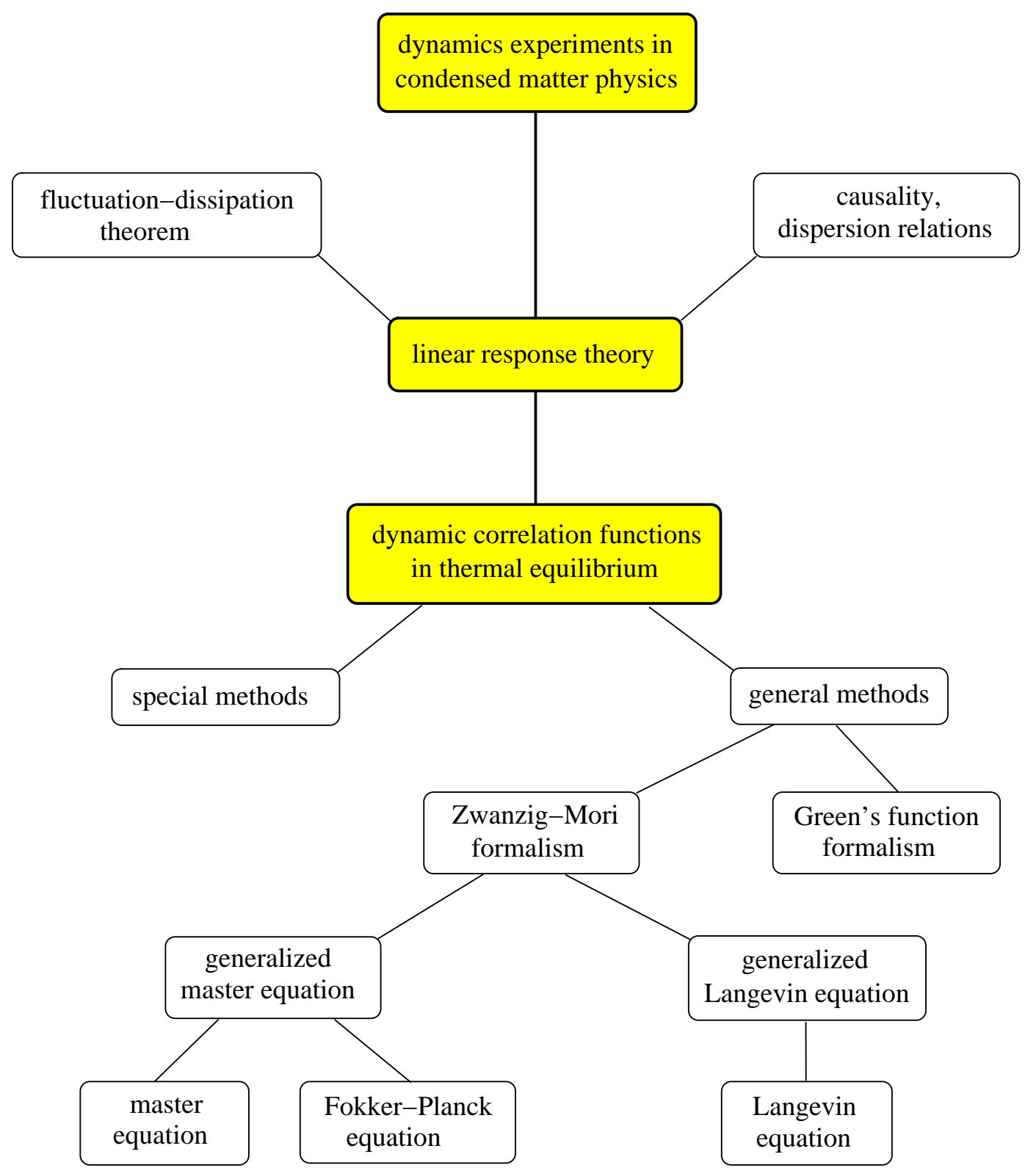




\section{Many-body system perturbed by radiation field ${ }_{[\mathrm{nnn} 25]}$}

Quantum many-body system in thermal equilibrium.

Hamiltonian: $\mathcal{H}_{0}$.

Density operator: $\rho_{0}=Z_{0}^{-1} e^{-\beta \mathcal{H}_{0}} \quad$ with $\beta=1 / k_{B} T, Z_{0}=\operatorname{Tr}\left[e^{-\beta \mathrm{H}_{0}}\right]$.

Dynamical variable: $A$ (describing some attribute of system).

Heisenberg equation of motion: $\frac{d A}{d t}=\frac{\imath}{\hbar}\left[\mathcal{H}_{0}, A\right]$.

Time evolution: $A(t)=e^{\imath \mathcal{H}_{0} t / \hbar} A e^{-\imath \mathcal{H}_{0} t / \hbar} \quad$ (formal solution).

Stationarity, $\left[\rho_{0}, \mathcal{H}_{0}\right]=0$, implies time-independent expectation values:

$$
\langle A(t)\rangle_{0}=\frac{1}{Z_{0}} \operatorname{Tr}\left[e^{-\beta \mathcal{H}_{0}} e^{\imath \mathcal{H}_{0} t / \hbar} A e^{-\imath \mathcal{H}_{0} t / \hbar}\right]=\frac{1}{Z_{0}} \operatorname{Tr}\left[e^{-\beta \mathcal{H}_{0}} A\right]=\text { const. }
$$

Time-dependent quantities do exist in thermal equilibrium!

Dynamic correlation function: $\langle A(t) A(0)\rangle_{0}=\frac{1}{Z_{0}} \operatorname{Tr}\left[e^{-\beta \mathcal{H}_{0}} e^{\imath \mathcal{H}_{0} t / \hbar} A e^{-\imath \mathcal{H}_{0} t / \hbar} A\right]$

In an experiment the system is necessarily perturbed:

$$
\mathcal{H}(t)=\mathcal{H}_{0}-b(t) B
$$

where $b(t)$ is some kind of radiation field (c-number) and $B$ is the dynamical system variable (operator) to which the field couples.

Examples:

\begin{tabular}{l|l}
$b(t)$ & $B$ \\
\hline magnetic field & magnetization \\
electric field & electric polarization \\
sound wave & mass density
\end{tabular}




\section{Linear response ${ }_{[\mathrm{nn} 26]}$}

Radiation field $b(t)$ perturbs equilibrium state of the system $\mathcal{H}_{0}$ via coupling to dynamical variable $B$.

System response to perturbation measured as expectation value of dynamical variable $A$.

Linear response to weak perturbations is predominant under most circumstances (away from criticality).

Response function $\tilde{\chi}_{A B}(t)$ (definition):

$$
\langle A(t)\rangle-\langle A\rangle_{0}=\int_{-\infty}^{\infty} d t^{\prime} \tilde{\chi}_{A B}\left(t-t^{\prime}\right) b\left(t^{\prime}\right) .
$$

- Linearity: $\tilde{\chi}_{A B}(t)$ is independent of $b(t)$.

- Hermiticity: $\tilde{\chi}_{A B}(t)$ is a real function.

- Causality: $\tilde{\chi}_{A B}(t)=0$ for $t<0$.

- Smoothness: $\left|\tilde{\chi}_{A B}(t)\right|<\infty$.

- Analyticity: $\tilde{\chi}_{A B}(t) \rightarrow 0$ for $t \rightarrow \infty$.

Generalized susceptibility (via Fourier transform):

$$
\chi_{A B}(\zeta)=\int_{-\infty}^{+\infty} d t e^{\imath \zeta t} \tilde{\chi}_{A B}(t) \quad \text { (analytic for } \Im\{\zeta\}>0 \text { ). }
$$

Complex function of real frequency:

$$
\chi_{A B}(\omega)=\lim _{\epsilon \rightarrow 0} \chi_{A B}(\omega+\imath \epsilon)=\chi_{A B}^{\prime}(\omega)+\imath \chi_{A B}^{\prime \prime}(\omega) .
$$

Linear response in frequency domain means no mixing of frequencies:

$$
\alpha(\omega)=\chi_{A B}(\omega) \beta(\omega),
$$

where

$$
\begin{gathered}
\tilde{\chi}_{A B}(t)=\int_{-\infty}^{+\infty} \frac{d \omega}{2 \pi} e^{-\imath \omega t} \chi_{A B}(\omega), \quad b(t)=\int_{-\infty}^{+\infty} \frac{d \omega}{2 \pi} e^{-\imath \omega t} \beta(\omega), \\
\langle A(t)\rangle-\langle A\rangle_{0}=\int_{-\infty}^{+\infty} \frac{d \omega}{2 \pi} e^{-\imath \omega t} \alpha(\omega) .
\end{gathered}
$$




\section{Kubo formula for response function}

Interaction representation for time evolution of $\mathcal{H}(t)=\mathcal{H}_{0}-b(t) B$ :

$$
\begin{gathered}
\frac{d A}{d t}=\frac{\imath}{\hbar}\left[\mathcal{H}_{0}, A\right] \Rightarrow A(t)=e^{\imath \mathcal{H}_{0} t / \hbar} A e^{-\imath \mathcal{H}_{0} t / \hbar}, \\
\frac{d B}{d t}=\frac{\imath}{\hbar}\left[\mathcal{H}_{0}, B\right] \Rightarrow B(t)=e^{\imath \mathcal{H}_{0} t / \hbar} B e^{-\imath \mathcal{H}_{0} t / \hbar}, \\
\frac{d \rho}{d t}=-\frac{\imath}{\hbar}[-b(t) B, \rho] \Rightarrow \rho(t)=\rho_{0}+\frac{\imath}{\hbar} \int_{-\infty}^{t} d t^{\prime} b\left(t^{\prime}\right)\left[B\left(t^{\prime}\right), \rho\left(t^{\prime}\right)\right] .
\end{gathered}
$$

Set $\rho(t)=\rho_{0}+\rho_{1}(t)$ with $\rho_{0}=Z_{0}^{-1} e^{-\beta \mathcal{H}_{0}}$.

Full response: $\langle A(t)\rangle-\langle A\rangle_{0}=\operatorname{Tr}\left\{\rho_{1}(t) A(t)\right\}$

Leading correction to $\rho_{0}: \rho_{1}(t) \simeq \frac{\imath}{\hbar} \int_{-\infty}^{t} d t^{\prime} b\left(t^{\prime}\right)\left[B\left(t^{\prime}\right), \rho_{0}\right]$

Linear response:

$$
\begin{aligned}
\langle A(t)\rangle-\langle A\rangle_{0} & =\frac{\imath}{\hbar} \int_{-\infty}^{t} d t^{\prime} b\left(t^{\prime}\right) \operatorname{Tr}\left\{\left[B\left(t^{\prime}\right), \rho_{0}\right] A(t)\right\} \\
& =\frac{\imath}{\hbar} \int_{-\infty}^{t} d t^{\prime} b\left(t^{\prime}\right) \operatorname{Tr}\left\{\rho_{0}\left[A(t), B\left(t^{\prime}\right)\right]\right\} \\
& =\frac{\imath}{\hbar} \int_{-\infty}^{t} d t^{\prime} b\left(t^{\prime}\right)\left\langle\left[A(t), B\left(t^{\prime}\right)\right]\right\rangle_{0} .
\end{aligned}
$$

Compare with definition of response function in $[\mathrm{nln} 26]$.

\section{Kubo formula:}

$$
\tilde{\chi}_{A B}\left(t-t^{\prime}\right)=\frac{\imath}{\hbar} \theta\left(t-t^{\prime}\right)\left\langle\left[A(t), B\left(t^{\prime}\right)\right]\right\rangle_{0} .
$$

- Causality requirement is ensured by step function $\theta\left(t-t^{\prime}\right)$.

- Hermitian $A, B$ imply Hermitian $\imath[A, B]$. Hence $\tilde{\chi}(t)$ is real.

- Linear response depends only on equilibrium quantities.

- Response function only depends on time difference $t-t^{\prime}$.

The Kubo formula establishes a general link between

- the dynamical properties of a many-body system at equilibrium,

- the dynamical response of that system to experimental probes. 


\section{Symmetry properties [nn30]}

Response function for Hermitian $A$ is real and vanishes for $t<0$ :

$$
\tilde{\chi}_{A A}(t)=\frac{\imath}{\hbar} \theta(t)\langle[A(t), A]\rangle=\tilde{\chi}_{A A}^{\prime}(t)+\imath \tilde{\chi}_{A A}^{\prime \prime}(t) .
$$

Reactive part is real and symmetric:

$$
\tilde{\chi}_{A A}^{\prime}(t)=\frac{1}{2}\left[\tilde{\chi}_{A A}(t)+\tilde{\chi}_{A A}(-t)\right]=\frac{\imath}{2 \hbar} \operatorname{sgn}(t)\langle[A(t), A]\rangle .
$$

Dissipative part is imaginary and antisymmetric:

$$
\tilde{\chi}_{A A}^{\prime \prime}(t)=\frac{1}{2 \imath}\left[\tilde{\chi}_{A A}(t)-\tilde{\chi}_{A A}(-t)\right]=\frac{1}{2 \hbar}\langle[A(t), A]\rangle .
$$

Response function is determined by its reactive or dissipative part alone:

$$
\tilde{\chi}_{A A}(t)=2 \theta(t) \tilde{\chi}_{A A}^{\prime}(t)=2 \imath \theta(t) \tilde{\chi}_{A A}^{\prime \prime}(t) .
$$

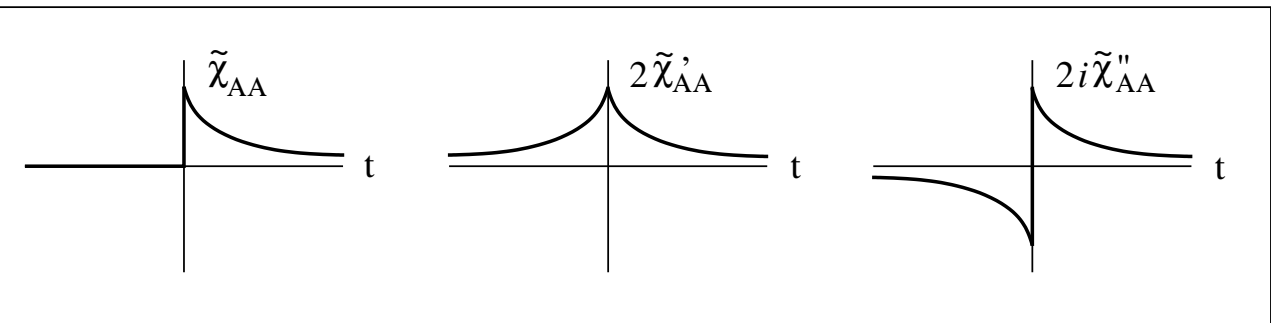

Generalized susceptibility is complex:

$$
\chi_{A A}(\omega)=\chi_{A A}^{\prime}(\omega)+\imath \chi_{A A}^{\prime \prime}(\omega) .
$$

Real part is symmetric:

$$
\chi_{A A}^{\prime}(\omega)=\frac{1}{2}\left[\chi_{A A}(\omega)+\chi_{A A}(-\omega)\right]=\chi_{A A}^{\prime}(-\omega) .
$$

Imaginary part is antisymmetric:

$$
\chi_{A A}^{\prime \prime}(\omega)=\frac{1}{2 \imath}\left[\chi_{A A}(\omega)-\chi_{A A}(-\omega)\right]=-\chi_{A A}^{\prime \prime}(-\omega) .
$$




\section{Kramers-Kronig dispersion relations ${ }_{[\mathrm{nn} 37]}$}

Use analyticity of $\chi_{A A}(\zeta)$ for $\Im\{\zeta\}>0$.

Cauchy integral: $\chi_{A A}(\zeta)=\frac{1}{2 \pi \imath} \int_{\mathcal{C}} d \zeta^{\prime} \frac{\chi_{A A}\left(\zeta^{\prime}\right)}{\zeta^{\prime}-\zeta}$

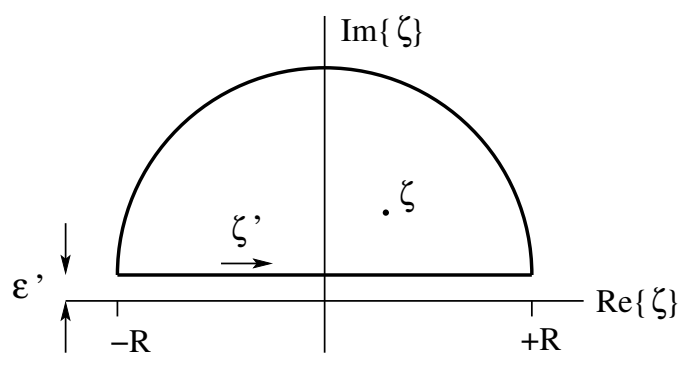

Integral converges for $\zeta^{\prime}=\omega^{\prime}+\imath \epsilon^{\prime}, \epsilon^{\prime} \rightarrow 0$.

Integral along semi-circle vanishes for $R \rightarrow \infty$ :

Sum rule implies $\chi_{A A}(\zeta) \lesssim|\zeta|^{-1}$ for $|\zeta| \rightarrow \infty$.

$$
\Rightarrow \chi_{A A}(\zeta)=\frac{1}{2 \pi \imath} \int_{-\infty}^{+\infty} d \omega^{\prime} \frac{\chi_{A A}\left(\omega^{\prime}\right)}{\omega^{\prime}-\zeta}
$$

Set $\zeta=\omega+\imath \epsilon$ and use $\lim _{\epsilon \rightarrow 0} \frac{1}{\omega^{\prime}-\omega \mp \imath \epsilon}=\mathrm{P} \frac{1}{\omega^{\prime}-\omega} \pm \imath \pi \delta\left(\omega^{\prime}-\omega\right)$.

$$
\begin{aligned}
\chi_{A A}(\omega) & =\lim _{\epsilon \rightarrow 0} \chi_{A A}(\omega+\imath \epsilon)=\lim _{\epsilon \rightarrow 0} \frac{1}{2 \pi \imath} \int_{-\infty}^{+\infty} d \omega^{\prime} \frac{\chi_{A A}\left(\omega^{\prime}\right)}{\omega^{\prime}-\omega-\imath \epsilon} \\
& =\frac{1}{2 \pi \imath} \mathrm{P} \int_{-\infty}^{+\infty} d \omega^{\prime} \frac{\chi_{A A}\left(\omega^{\prime}\right)}{\omega^{\prime}-\omega}+\underbrace{\frac{1}{2} \int_{-\infty}^{+\infty} d \omega^{\prime} \chi_{A A}\left(\omega^{\prime}\right) \delta\left(\omega^{\prime}-\omega\right)}_{\frac{1}{2} \chi_{A A}(\omega)} . \\
\Rightarrow & \chi_{A A}(\omega) \doteq \chi_{A A}^{\prime}(\omega)+\imath \chi_{A A}^{\prime \prime}(\omega)=\frac{1}{\pi \imath} \mathrm{P} \int_{-\infty}^{+\infty} d \omega^{\prime} \frac{\chi_{A A}\left(\omega^{\prime}\right)}{\omega^{\prime}-\omega} .
\end{aligned}
$$

Consider real and imaginary parts of this relation separately:

$$
\chi_{A A}^{\prime}(\omega)=\frac{1}{\pi} \mathrm{P} \int_{-\infty}^{+\infty} d \omega^{\prime} \frac{\chi_{A A}^{\prime \prime}\left(\omega^{\prime}\right)}{\omega^{\prime}-\omega}, \quad \chi_{A A}^{\prime \prime}(\omega)=-\frac{1}{\pi} \mathrm{P} \int_{-\infty}^{+\infty} d \omega^{\prime} \frac{\chi_{A A}^{\prime}\left(\omega^{\prime}\right)}{\omega^{\prime}-\omega} .
$$

The Kramers-Kronig relations are a consequence of the causality property of the response function. 


\section{[nex63] Causality property of response function.}

The Kramers-Kronig dispersion relations

$$
\chi_{A A}^{\prime}(\omega)=\frac{1}{\pi} \int_{-\infty}^{\infty} d \omega^{\prime} \frac{\chi_{A A}^{\prime \prime}\left(\omega^{\prime}\right)}{\omega^{\prime}-\omega}, \quad \chi_{A A}^{\prime \prime}(\omega)=-\frac{1}{\pi} \int_{-\infty}^{\infty} d \omega^{\prime} \frac{\chi_{A A}^{\prime}\left(\omega^{\prime}\right)}{\omega^{\prime}-\omega}
$$

between the reactive part $\chi_{A A}^{\prime}(\omega)$ and the dissipative part $\chi_{A A}^{\prime \prime}(\omega)$ of the generalized susceptibility $\chi_{A A}(\omega)$ are a direct consequence of the causality property of the response function $\tilde{\chi}_{A A}(t)$. Show that $\chi_{A A}(\zeta)$ for $\Im(\zeta)>0$ can be expressed in terms of $\chi_{A A}^{\prime \prime}(\omega)$ as follows:

$$
\chi_{A A}(\zeta)=\frac{1}{\pi} \int_{-\infty}^{\infty} d \omega \frac{\chi_{A A}^{\prime \prime}(\omega)}{\omega-\zeta} .
$$

\section{Solution:}




\section{Energy transfer ${ }_{[\ln 338]}$}

Hamiltonian of system and interaction with radiation field:

$$
\mathcal{H}(t)=\mathcal{H}_{0}+\mathcal{H}_{1}(t)=\mathcal{H}_{0}-a(t) A
$$

Interaction between system and radiation field involves energy transfer.

Rate at which average energy of system changes:

$$
\frac{d}{d t}\left\langle\mathcal{H}_{0}\right\rangle=\frac{1}{\imath \hbar}\left\langle\left[\mathcal{H}_{0}, \mathcal{H}(t)\right]\right\rangle=-\frac{1}{\imath \hbar} a(t)\left\langle\left[\mathcal{H}_{0}, A(t)\right]\right\rangle .
$$

Calculate linear response $\left\langle\left[\mathcal{H}_{0}, A(t)\right]\right\rangle-\underbrace{\left\langle\left[\mathcal{H}_{0}, A\right]\right\rangle_{0}}_{0} \cdot{ }^{1}$

Application of Kubo formula [nln27]:

$$
\begin{gathered}
\left\langle\left[\mathcal{H}_{0}, A(t)\right]\right\rangle=\frac{\imath}{\hbar} \int_{-\infty}^{t} d t^{\prime} a\left(t^{\prime}\right)\left\langle\left[\left[\mathcal{H}_{0}, A(t)\right], A\left(t^{\prime}\right)\right]\right\rangle_{0} . \\
\Rightarrow \frac{d}{d t}\left\langle\mathcal{H}_{0}\right\rangle=-\frac{1}{\hbar^{2}} a(t) \int_{-\infty}^{t} d t^{\prime} a\left(t^{\prime}\right)\left\langle[\overbrace{\left[\mathcal{H}_{0}, A(t)\right]}^{-\imath \hbar d A / d t}, A\left(t^{\prime}\right)]\right\rangle_{0} \\
= \\
\frac{\imath}{\hbar} a(t) \int_{-\infty}^{t} d t^{\prime} a\left(t^{\prime}\right) \frac{\partial}{\partial t}\left\langle\left[A(t), A\left(t^{\prime}\right)\right]\right\rangle_{0} \\
=\int_{-\infty}^{+\infty} d t^{\prime} a(t) a\left(t^{\prime}\right) \frac{\partial}{\partial t} \tilde{\chi}_{A A}\left(t-t^{\prime}\right)
\end{gathered}
$$

with response function

$$
\tilde{\chi}_{A A}\left(t-t^{\prime}\right)=\frac{\imath}{\hbar} \theta\left(t-t^{\prime}\right)\left\langle\left[A(t), A\left(t^{\prime}\right)\right]\right\rangle_{0} .
$$

The time-averaged energy transfer depends only on the absorptive part, $\chi_{A A}^{\prime \prime}(\omega)$, of the generalized susceptibility as demonstrated in [nex64] for a monochromatic perturbation.

\footnotetext{
${ }^{1}$ We have $\left\langle\left[\mathcal{H}_{0}, A\right]\right\rangle_{0}=\operatorname{Tr}\left\{e^{-\beta \mathcal{H}_{0}} \mathcal{H}_{0} A-e^{-\beta \mathcal{H}_{0}} A \mathcal{H}_{0}\right\} / Z_{0}=0$ in thermal equilibrium.
} 


\section{[nex64] Reactive and absorptive parts of linear response.}

In the framework of linear response theory for $H=H_{0}-a(t) A$, the rate of energy transfer between the system and the radiation field is

$$
\frac{d}{d t}\left\langle H_{0}\right\rangle=\int_{-\infty}^{\infty} d t^{\prime} a(t) a\left(t^{\prime}\right) \frac{\partial}{\partial t} \tilde{\chi}_{A A}\left(t-t^{\prime}\right)
$$

where

$$
\tilde{\chi}_{A A}\left(t-t^{\prime}\right)=\frac{i}{\hbar} \theta\left(t-t^{\prime}\right)\left\langle\left[A(t), A\left(t^{\prime}\right)\right]\right\rangle_{0}
$$

is the Kubo formula for the response function (see [nln38].)

(a) Evaluate this expression for a monochromatic perturbation,

$$
a(t)=\frac{1}{2} a_{m}\left(e^{i \omega_{0} t}+e^{-i \omega_{0} t}\right)
$$

and express it in terms of the reactive part, $\chi_{A A}^{\prime}(\omega)$, and the absorptive (dissipative) part, $\chi_{A A}^{\prime \prime}(\omega)$, of the generalized susceptibility $\chi_{A A}(\omega)$ as defined in [nln26].

(b) Show that the time-averaged energy transfer depends only on the absorptive part of $\chi_{A A}(\omega)$ :

$$
\overline{\frac{d}{d t}\left\langle H_{0}\right\rangle}=\frac{1}{2} a_{m}^{2} \omega_{0} \chi_{A A}^{\prime \prime}\left(\omega_{0}\right) .
$$

\section{Solution:}




\section{Fluctuation-dissipation theorem [nn339]}

Three dynamical quantities in time domain: ${ }^{1}$

$$
\begin{array}{ll}
\triangleright \quad \tilde{\chi}_{A A}^{\prime \prime}(t) \doteq \frac{1}{2 \hbar}\left\langle[A(t), A]_{-}\right\rangle \quad \text { response function (dissipative part), } \\
\triangleright \quad \tilde{\Phi}_{A A}(t) \doteq \frac{1}{2}\left\langle[A(t), A]_{+}\right\rangle-\langle A\rangle^{2} \quad \text { fluctuation function, } \\
\triangleright \quad \tilde{S}_{A A}(t) \doteq\langle A(t) A\rangle-\langle A\rangle^{2} \quad \text { correlation function. }
\end{array}
$$

Relations:

$$
\tilde{\chi}_{A A}^{\prime \prime}(t)=\frac{1}{2 \hbar}\left[\tilde{S}_{A A}(t)-\tilde{S}_{A A}(-t)\right], \quad \tilde{\Phi}_{A A}(t)=\frac{1}{2}\left[\tilde{S}_{A A}(t)+\tilde{S}_{A A}(-t)\right] .
$$

Transformation properties under time reversal (for real $t$ ):

- $\tilde{\chi}_{A A}^{\prime \prime}(-t)=-\tilde{\chi}_{A A}^{\prime \prime}(t)=\left[\tilde{\chi}_{A A}^{\prime \prime}(t)\right]^{*} \quad$ imaginary and antisymmetric,

- $\tilde{\Phi}_{A A}(-t)=\tilde{\Phi}_{A A}(t)=\left[\tilde{\Phi}_{A A}(t)\right]^{*} \quad$ real and symmetric,

- $\tilde{S}_{A A}(-t)=\tilde{S}_{A A}(t-\imath \hbar \beta)=\left[\tilde{S}_{A A}(t)\right]^{*}$ complex. $^{2}$

To make the last symmetry relation more transparent we write

$$
\begin{aligned}
\langle A(-t) A\rangle & =\operatorname{Tr}\left[e^{-\beta \mathcal{H}_{0}} e^{-\imath \mathcal{H}_{0} t / \hbar} A e^{\imath \mathcal{H}_{0} t / \hbar} A\right] \\
& =\operatorname{Tr}\left[e^{-\beta \mathcal{H}_{0}} e^{\imath \mathcal{H}_{0}(t-\imath \hbar \beta) / \hbar} A e^{-\imath \mathcal{H}_{0}(t-\imath \hbar \beta) / \hbar} A\right]=\langle A(t-\imath \beta \hbar) A\rangle .
\end{aligned}
$$

The imaginary part of the correlation function vanishes if

- if $\beta=0$ i.e. at infinite temperature,

- if $\hbar=0$ i.e. for classical systems.

\footnotetext{
${ }^{1}$ using $[,]_{-}$for commutators and $[,]_{+}$for anti-commutators.

${ }^{2}$ with symmetric real part and antisymmetric imaginary part.
} 
Three dynamical quantities in frequency domain:

$$
\begin{array}{lll}
\triangleright & \chi_{A A}^{\prime \prime}(\omega) \doteq \int_{-\infty}^{+\infty} d t e^{\imath \omega t} \tilde{\chi}_{A A}^{\prime \prime}(t) & \text { dissipation function, } \\
\triangleright & \Phi_{A A}(\omega) \doteq \int_{-\infty}^{+\infty} d t e^{\imath \omega t} \tilde{\Phi}_{A A}(t) & \text { spectral density, } \\
\triangleright & S_{A A}(\omega) \doteq \int_{-\infty}^{+\infty} d t e^{\imath \omega t} \tilde{S}_{A A}(t) & \text { structure function. }
\end{array}
$$

Symmetry properties:

- $\chi_{A A}^{\prime \prime}(-\omega)=-\chi_{A A}^{\prime \prime}(\omega) \quad$ real and antisymmetric,

- $\Phi_{A A}(-\omega)=\Phi_{A A}(\omega) \quad$ real and symmetric,

- $S_{A A}(-\omega)=e^{-\beta \hbar \omega} S_{A A}(\omega) \quad$ real and satisfying detailed balance.

Relations:

$$
\chi_{A A}^{\prime \prime}(\omega)=\frac{1}{2 \hbar}\left(1-e^{-\beta \hbar \omega}\right) S_{A A}(\omega), \quad \Phi_{A A}(\omega)=\frac{1}{2}\left(1+e^{-\beta \hbar \omega}\right) S_{A A}(\omega) .
$$

Fluctuation-dissipation relation (general quantum version):

$$
\Phi_{A A}(\omega)=\hbar \operatorname{coth}\left(\frac{1}{2} \beta \hbar \omega\right) \chi_{A A}^{\prime \prime}(\omega)
$$

Dissipation effects from an interaction with a weak external force as encoded in $\chi_{A A}^{\prime \prime}(\omega)$ are determined by natural fluctuations existing in thermal equilibrium as encoded in $\Phi_{A A}(\omega)$.

Classical limit (no zero-point fluctuations):

$$
\Phi_{A A}(\omega)_{c l} \stackrel{\hbar \rightarrow 0}{\longrightarrow} \frac{2 k_{B} T}{\omega} \chi_{A A}^{\prime \prime}(\omega) .
$$

Classical fluctuations of any frequency related to static susceptibility:

$$
\begin{aligned}
\left\langle(A-\langle A\rangle)^{2}\right\rangle & =\tilde{\phi}_{A A}(t=0)=\lim _{t \rightarrow 0} \int_{-\infty}^{+\infty} \frac{d \omega}{2 \pi} e^{-\imath \omega t} \Phi_{A A}(\omega) \\
& =k_{B} T \int_{-\infty}^{+\infty} \frac{d \omega}{\pi} \omega^{-1} \chi_{A A}^{\prime \prime}(\omega)=k_{B} T \lim _{\omega^{\prime} \rightarrow 0} \frac{1}{\pi} \int_{-\infty}^{+\infty} d \omega \frac{\chi_{A A}^{\prime \prime}(\omega)}{\omega-\omega^{\prime}} \\
& =k_{B} T \chi_{A A}^{\prime}\left(\omega^{\prime}=0\right)=k_{B} T \chi_{A A}\left(\omega^{\prime}=0\right) \doteq k_{B} T \chi_{A A} .
\end{aligned}
$$




\section{Moment Expansion [nnt78]}

Correlation function and structure function:

$$
\tilde{S}_{A A}(t) \doteq\langle A(t) A\rangle-\langle A\rangle^{2}=\int_{-\infty}^{+\infty} \frac{d \omega}{2 \pi} e^{-\imath \omega t} S_{A A}(\omega)=\sum_{n=0}^{\infty} \tilde{M}_{n} \frac{(-\imath t)^{n}}{n !} .
$$

Frequency moments: use $\dot{\tilde{S}}_{A A}(t)=\langle\dot{A}(t) A\rangle=(-\imath / \hbar)\langle[A(t), \mathcal{H}] A\rangle$.

$\left.\left.\left.\tilde{M}_{n} \doteq \int_{-\infty}^{+\infty} \frac{d \omega}{2 \pi} \omega^{n} S_{A A}(\omega)=\imath^{n}\left[\frac{d^{n}}{d t^{n}} \tilde{S}_{A A}(t)\right]_{t=0}=\hbar^{-n}\langle\underbrace{[[\cdots[}_{n} A, \mathcal{H}], \mathcal{H}\right], \cdots, \mathcal{H}\right] A\right\rangle$,

High-temperature limit $T \rightarrow \infty$ :

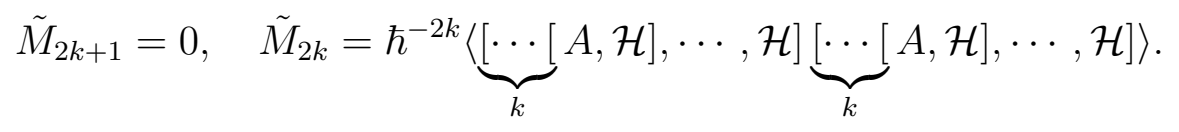

Classical limit $\hbar \rightarrow 0$ : use $\quad \dot{\tilde{S}}_{A A}(t)=\langle\dot{A}(t) A\rangle=\langle\{A(t), \mathcal{H}\} A\rangle$.

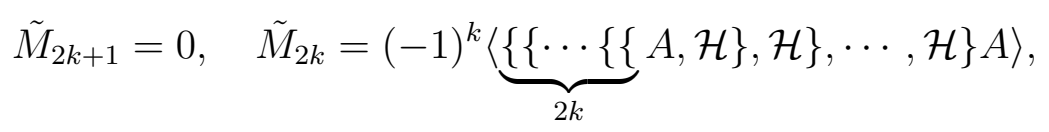

Fluctuation function:

$$
\begin{aligned}
& \tilde{\Phi}_{A A}(t) \doteq \frac{1}{2}\left\langle[A(t), A]_{+}\right\rangle-\langle A\rangle^{2}=\sum_{k=0}^{\infty} \tilde{M}_{2 k} \frac{(-\imath t)^{2 k}}{(2 k) !} \\
& \left.\left.\left.\left.\tilde{M}_{2 k}=\frac{1}{2 \hbar^{2 k}}\langle\underbrace{[[\cdots[[}_{2 k} A, \mathcal{H}], \mathcal{H}\right], \cdots, \mathcal{H}\right] A\right]_{+}\right\rangle .
\end{aligned}
$$

Dissipation function:

$$
\begin{gathered}
\tilde{\chi}_{A A}^{\prime \prime}(t) \doteq \frac{1}{2 \hbar}\langle[A(t), A]\rangle=\hbar^{-1} \sum_{k=0}^{\infty} \tilde{M}_{2 k+1} \frac{(-\imath t)^{2 k+1}}{(2 k+1) !} \\
\left.\left.\left.\left.\tilde{M}_{2 k+1}=\frac{1}{2 \hbar^{2 k+1}}\langle\underbrace{\langle[\cdots[}_{2 k+1} A, \mathcal{H}], \mathcal{H}\right], \cdots, \mathcal{H}\right] A\right]\right\rangle .
\end{gathered}
$$

Moment expansion not guaranteed to converge.

Convergence problem may be circumnavigated by recursion method. 


\section{[nex65] Spectral representation of dynamical quantities.}

Consider a quantum Hamiltonian system with known eigenvalues and eigenvectors,

$$
H|n\rangle=E_{n}|n\rangle, \quad n=0,1, \ldots,
$$

in thermal equilibrium at temperature $T$. Express (a) the structure function $S_{A A}(\omega)$, (b) the spectral density $\Phi_{A A}(\omega)$, (c) the dissipation function $\chi_{A A}^{\prime \prime}(\omega)$, and $(\mathrm{d})$ the generalized susceptibility $\chi_{A A}(\omega+i \epsilon)$, all defined in [nln39], in terms of the temperature parameter $\beta=1 / k_{B} T$, the energy levels $E_{n}$, and the matrix elements $\langle n|A| m\rangle$. For simplicity assume that $\langle A\rangle \doteq Z^{-1} \operatorname{Tr}\left[e^{-\beta H} A\right]=0$. The last result reads

$$
\chi_{A A}(\omega+i \epsilon)=\frac{1}{Z} \sum_{m, n}\left(e^{-\beta E_{m}}-e^{-\beta E_{n}}\right) \frac{|\langle n|A| m\rangle|^{2}}{\hbar \omega-\left(E_{m}-E_{n}\right)+i \epsilon} .
$$

\section{Solution:}




\section{[nex66] Linear response of classical relaxator.}

The classical relaxator is defined by the equation of motion,

$$
\dot{x}+\frac{1}{\tau_{0}} x=a(t)
$$

where $\tau_{0}$ represents a relaxation time and $a(t)$ a weak periodic perturbation. The (linear) response function is extracted from the relation

$$
\langle x(t)\rangle-\langle x\rangle_{0}=\int_{-\infty}^{t} d t^{\prime} \tilde{\chi}_{x x}\left(t-t^{\prime}\right) a\left(t^{\prime}\right),
$$

where $x(t)$ is the solution of (1).

(a) Solve (1) formally as in [nex53] and compare the result with (2) to show that the response function must be

$$
\tilde{\chi}_{x x}(t)=e^{-t / \tau_{0}} \theta(t) .
$$

(b) Calculate the generalized susceptibility $\chi_{x x}(\omega)$ via Fourier analysis of (1) as in [nex119]. Show that the Fourier transform of (3) yields the same result, namely

$$
\chi_{x x}(\omega)=\frac{\tau_{0}}{1-i \omega \tau_{0}} .
$$

(c) Extract from $\chi_{x x}(\omega)$ its reactive part $\chi_{x x}^{\prime}(\omega)$ and its dissipative part $\chi_{x x}^{\prime \prime}(\omega)$ as prescribed in [nln30] and verify their symmtry properties.

(d) Use the (classical) fluctuation-dissipation theorem from [nln39] to infer the spectral density $\Phi_{x x}(\omega)$ from the dissipation function $\chi_{x x}^{\prime \prime}(\omega)$.

(e) Retrieve from the generalized susceptibility (4) the response function (3) via inverse Fourier transform carried out as a contour integral.

(f) Retrieve $\chi_{x x}^{\prime}(\omega)$ from $\chi_{x x}^{\prime \prime}(\omega)$ and vice versa via a numerical principal-value integration of the Kramers-Kronig relations as stated in [nln37]. Use $\tau=1$ and consider the interval $-2 \leq \omega \leq 2$. Plot the curves obtained via integration for comparison with the analytic expressions. Integrate over the intervals $-\infty<\omega^{\prime}<\omega-\epsilon$ and $\omega+\epsilon<\omega^{\prime}<+\infty$ with $0<\epsilon \ll 1$.

\section{Solution:}




\section{Dielectric Relaxation in Liquid Water ${ }_{[\ln n \pi]}$}

- $\mathrm{H}_{2} \mathrm{O}$ molecules have permanent electric dipole moment (polar molecules.)

- Alignment of dipole moments with external electric field $\mathbf{E}$ is energetically favorable.

- Alignment tendency is counteracted by thermal fluctuations.

- Turning $\mathbf{E}$ on/off initiates relaxation process toward equilibrium.
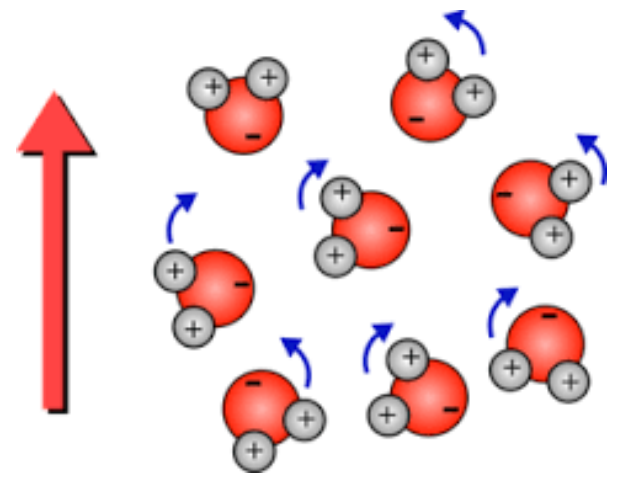

- $P(t)$ : instantaneous electric polarization (average dipole moment)

- $\chi_{0}$ : static dielectric susceptibility

- $\tau_{0}$ : characteristic relaxation time

- $E(t)$ : oscillating electric field

- $\frac{d}{d t} P(t)=-\frac{1}{\tau_{0}}\left[P(t)-\chi_{0} E(t)\right]:$ dielectric relaxation process

- $\langle P\rangle=\chi_{0} E$ : static (linear) response

- $\chi_{P P}(\omega)=\frac{\chi_{0}}{\tau_{0}} \chi_{x x}(\omega)$ : link to classical relaxator [nex66]

- $\langle P(t) P\rangle-\langle P\rangle^{2}=k_{B} T \chi_{0} e^{-t / \tau_{0}}$ : correlation fct. (from fluc.-diss. rel.)

- $\left\langle P^{2}\right\rangle \doteq \frac{1}{3} n p_{0}^{2}=k_{B} T \chi_{0}$ : zero-field limit

- $n$ : number density of molecules

- $p_{0}$ : permanent molecular electric dipole moment

- $\chi_{0}(T)=\frac{n p_{0}^{2}}{3 k_{B} T}: T$-dependence of dielectric susceptibility 


\section{[nex67] Linear response of classical oscillator.}

The classical oscillator is defined by the equation of motion,

$$
m \ddot{x}+\gamma \dot{x}+m \omega_{0}^{2} x=a(t),
$$

where $\gamma$ is the attenuation coefficient, $m \omega_{0}^{2}$ the spring constant, and $a(t)$ a weak periodic perturbation. The (linear) response function is defined by the relation

$$
\langle x(t)\rangle-\langle x\rangle_{0}=\int_{-\infty}^{t} d t^{\prime} \tilde{\chi}_{x x}\left(t-t^{\prime}\right) a\left(t^{\prime}\right),
$$

where $x(t)$ is the solution of (1).

(a) Calculate the generalized susceptibility $\chi_{x x}(\omega)$ as well as its reactive part $\chi_{x x}^{\prime}(\omega)$ and its dissipative part $\chi_{x x}^{\prime \prime}(\omega)$.

(b) Use the (classical) fluctuation-dissipation theorem to infer the spectral density $\Phi_{x x}(\omega)$ from the dissipation function $\chi_{x x}^{\prime \prime}(\omega)$.

\section{Solution:}




\section{Dynamic Structure Factor [nns8]}

Inelastic scattering of particles (electrons, neutrons, photons,...) involves momentum transfer, $\hbar \mathbf{q}=\hbar \mathbf{k}_{\mathrm{f}}-\hbar \mathbf{k}_{\mathrm{i}}$, and energy transfer, $\hbar \omega=E_{\mathrm{f}}-E_{\mathrm{i}}$, between scattered particles and collective excitations in the system.

Scattering cross section is proportional to dynamic structure factor:

$$
\frac{d^{2} \sigma}{d \omega d \Omega} \propto S_{A A}(\mathbf{q}, \omega)
$$

Target system: $\mathcal{H}_{0}|\lambda\rangle=E_{\lambda}|\lambda\rangle$.

Interaction with scattering radiation: $A(\mathbf{q}, t)=\int d^{3} r e^{-\imath \mathbf{k}_{\mathrm{i}} \cdot \mathbf{r}} V(\mathbf{r}, t) e^{\imath \mathbf{k}_{\mathrm{f}} \cdot \mathbf{r}}$.

Scattering events produce transitions $|\lambda\rangle \rightarrow\left|\lambda^{\prime}\right\rangle$ in target system.

Transition rates: $T(\mathbf{q}, \omega)=\left|\left\langle\lambda|A(\mathbf{q})| \lambda^{\prime}\right\rangle\right|^{2} \delta\left(\hbar \omega-E_{\lambda^{\prime}}+E_{\lambda}\right) \delta_{\mathbf{q}-\mathbf{k}_{\lambda^{\prime}}+\mathbf{k}_{\lambda}+\mathbf{Q}}$.

Dynamic structure factor: $S_{A A}(\mathbf{q}, \omega)=\frac{2 \pi}{Z} \sum_{\lambda, \lambda^{\prime}} e^{-\beta E_{\lambda}} T(\mathbf{q}, \omega)$.

Electron scattering (Coulomb interaction with target charge density):

$$
V(\mathbf{r}, t)=\frac{e \rho(\mathbf{R}, t)}{|\mathbf{r}-\mathbf{R}|} \Rightarrow S_{\rho \rho}(\mathbf{q}, \omega)=\int_{-\infty}^{+\infty} d t e^{\imath \omega t}\langle\rho(\mathbf{q}, t) \rho(-\mathbf{q}, 0)\rangle
$$

Nuclear neutron scattering (contact interaction with target particle density):

$$
V(\mathbf{r}, t)=a \delta(\mathbf{r}-\mathbf{R}) n(\mathbf{R}, t) \quad \Rightarrow \quad S_{n n}(\mathbf{q}, \omega)=\int_{-\infty}^{+\infty} d t e^{\imath \omega t}\langle n(\mathbf{q}, t) n(-\mathbf{q}, 0)\rangle
$$

Magnetic neutron scattering (interaction with target magnetisation):

$$
\begin{aligned}
V(\mathbf{r}, t) & =S_{\mu}(\mathbf{r}) V_{\mu \nu}(\mathbf{r}-\mathbf{R}) M_{\nu}(\mathbf{R}, t) \\
\Rightarrow S_{\mu \nu}(\mathbf{q}, \omega) & =\int_{-\infty}^{+\infty} d t e^{\imath \omega t}\left\langle M_{\mu}(\mathbf{q}, t) M_{\nu}(-\mathbf{q}, 0)\right\rangle .
\end{aligned}
$$

Light scattering (interaction with inhomogeneities in dielectric function):

$$
\epsilon(\mathbf{r}, t) \Rightarrow S_{\epsilon \epsilon}(\mathbf{q}, \omega)=\int_{-\infty}^{+\infty} d t e^{\imath \omega t}\langle\epsilon(\mathbf{q}, t) \epsilon(-\mathbf{q}, 0)\rangle
$$




\section{Scattering from Free Atoms [nnmas]}

Consider a dilute gas of atoms with mass $M$. Interaction between gas atoms limited to (rare) collisions.

Hamiltonian: $\mathcal{H}=\frac{p^{2}}{2 M} \quad$ (dominated by kinetic energy).

Contact interaction between gas atom at position $\mathbf{R}(t)$ and scattering radiation (see [nln89]) defines dynamical variable relevant for scattering process:

$$
A(\mathbf{q}, t)=\int d^{3} r e^{\imath \mathbf{q} \cdot \mathbf{r}} \delta(\mathbf{r}-\mathbf{R}(t))=e^{\imath \mathbf{q} \cdot \mathbf{R}(t)} .
$$

Equation of motion (setting $\hbar \equiv 1):^{1}$

$$
\imath \frac{\partial A}{\partial t}=[A, \mathcal{H}]=\frac{1}{2 M}\left[e^{\imath \mathbf{q} \cdot \mathbf{R}}, p^{2}\right]=-A \frac{1}{2 M}\left(2 \mathbf{q} \cdot \mathbf{p}+q^{2}\right) .
$$

Formal solution:

$$
A(\mathbf{q}, t)=e^{\imath \mathbf{q} \cdot \mathbf{R}(0)} \exp \left(\frac{\imath t\left(2 \mathbf{q} \cdot \mathbf{p}+q^{2}\right)}{2 M}\right)
$$

Correlation function: $\tilde{S}_{A A}(\mathbf{q}, t) \doteq\left\langle A^{\dagger}(\mathbf{q}, t) A(\mathbf{q}, 0)\right\rangle$.

$$
\begin{aligned}
\Rightarrow \tilde{S}_{A A}(\mathbf{q}, t) & \doteq e^{-\imath t q^{2} / 2 M}\langle\exp (-\imath t \mathbf{q} \cdot \mathbf{p} / M)\rangle \\
& =e^{-\imath t q^{2} / 2 M} \frac{1}{Z} \int d^{3} p e^{-\beta p^{2} / 2 M} e^{-\imath t \mathbf{q} \cdot \mathbf{p} / M} \\
& =e^{-\imath t q^{2} / 2 M} \frac{1}{Z} \underbrace{\int d^{3} p \exp \left(\frac{(\sqrt{\beta} \mathbf{p}+\imath t \mathbf{q} / \sqrt{\beta})^{2}}{2 M}\right)}_{Z} e^{-q^{2} t^{2} / 2 M \beta} \\
& =\exp \left(-\frac{q^{2}\left(t^{2} / \beta+\imath t\right)}{2 M}\right) .
\end{aligned}
$$

Third line: Gaussian integral is unaffected by a constant shift in $\mathbf{p}$.

Note symmetry property from $[\mathrm{n} \ln 39]: \tilde{S}_{A A}(\mathbf{q},-t)=\tilde{S}_{A A}(\mathbf{q}, t-\imath \beta)$.

${ }^{1}$ Use $[\mathbf{R}, \mathbf{p}]=\imath,[A, \mathbf{p}]=-\mathbf{q} A,\left[A, p^{2}\right]=[A, \mathbf{p}] \cdot \mathbf{p}+\mathbf{p} \cdot[A, \mathbf{p}]=-A \mathbf{q} \cdot \mathbf{p}-\mathbf{p} \cdot \mathbf{q} A$, $A \mathbf{q} \cdot \mathbf{p}-\mathbf{p} \cdot \mathbf{q} A=-A q^{2}, \Rightarrow\left[A, p^{2}\right]=-A\left(2 \mathbf{q} \cdot \mathbf{p}+q^{2}\right)$. 
Dynamic structure factor via Fourier transform:

$$
\begin{aligned}
S_{A A}(\mathbf{q}, \omega) & \doteq \int_{-\infty}^{+\infty} d t e^{\imath \omega t} \tilde{S}_{A A}(\mathbf{q}, t) \\
& =\sqrt{\frac{2 \pi M \beta}{q^{2}}} \exp \left(-\frac{M \beta}{2 q^{2}}\left[\omega-q^{2} / 2 M\right]^{2}\right) .
\end{aligned}
$$

- Scattering is isotropic, only dependent on magnitude of $\mathbf{q}$.

- Maximum intensity occurs when energy transfer $\omega$ and momentum transfer $\mathbf{q}$ reflect energy momentum relation, $\omega=q^{2} / 2 M$, of free, nonrelativistic gas particle.

- Lineshape broadens with increasing temperature and/or decreasing mass of gas atoms.

- Note detailed-balance condition from [nln39]:

$$
S_{A A}(\mathbf{q},-\omega)=e^{-\beta \omega} S_{A A}(\mathbf{q}, \omega) .
$$

- In the limit $M \rightarrow \infty$ at fixed temperature, the atoms slow down and come to rest. The scattering becomes elastic in nature, still isotropic and with zero energy transfer:

$$
S_{A A}(\mathbf{q}, \omega) \stackrel{M \rightarrow \infty}{\longrightarrow} 2 \pi \delta(\omega) .
$$

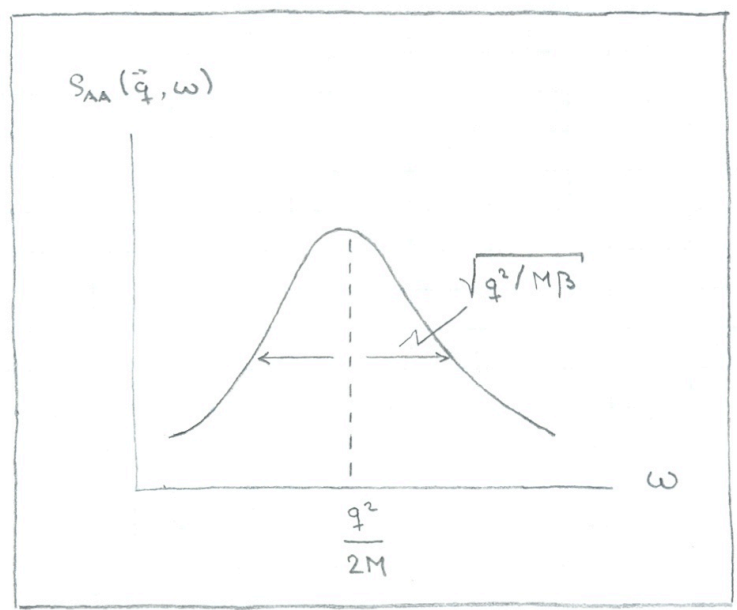




\section{Scattering from Atoms Bound to Lattice [nnn94]}

Consider array of atoms harmonically bound to sites of rigid lattice. We set $\hbar=1$ and atomic mass $M=1$ :

Hamiltonian: $\mathcal{H}=\frac{1}{2}\left(p^{2}+\omega_{0}^{2} u^{2}\right)=\omega_{0}\left(a^{\dagger} a+\frac{1}{2}\right)$.

Displacement of atom from equilibrium position: ${ }^{1} u(t)=\frac{1}{\sqrt{2 \omega_{0}}}\left(a e^{-\imath \omega_{0} t}+a^{\dagger} e^{\imath \omega_{0} t}\right)$.

Dynamical variable: $A(q, t)=e^{\imath q u(t)}$.

Correlation function: $\tilde{S}_{A A}(q, t) \doteq\left\langle A^{\dagger}(q, 0) A(q,-t)\right\rangle$.

Use Baker-Hausdorff expansion: ${ }^{2} e^{A} e^{B}=\exp \left(A+B+\frac{1}{2}[A, B]+\ldots\right)$.

$$
\begin{aligned}
\Rightarrow \tilde{S}_{A A}(q, t) & =\left\langle e^{-\imath q u} e^{\imath q u(-t)}\right\rangle=\left\langle e^{\imath q[u(-t)-u]}\right\rangle e^{q^{2}[u, u(-t)] / 2} \\
& =e^{-q^{2}\left\langle[u(-t)-u]^{2} / 2\right\rangle} e^{q^{2}[u u(-t)-u(-t) u] / 2}=e^{-q^{2}\left[\left\langle u^{2}\right\rangle-\langle u u(-t)\rangle\right]} .
\end{aligned}
$$

Boson distribution: $\left\langle a^{\dagger} a\right\rangle=n_{\mathrm{B}}=\frac{1}{e^{\beta \omega_{0}}-1} \Rightarrow\left\langle a a^{\dagger}\right\rangle=1+n_{\mathrm{B}}$.

Debye-Waller factor: $W=\frac{1}{2} q^{2}\left\langle u^{2}\right\rangle=\frac{q^{2}}{4 \omega_{0}} \underbrace{\operatorname{coth} \frac{\beta \omega_{0}}{2}}_{1+2 n_{\mathrm{B}}}, \Rightarrow e^{-q^{2}\left\langle u^{2}\right\rangle} \doteq e^{-2 W}$.

$$
\begin{aligned}
\langle u u(-t)\rangle & =\frac{1}{2 \omega_{0}}\left[\left\langle a^{\dagger} a\right\rangle e^{\imath \omega_{0} t}+\left\langle a a^{\dagger}\right\rangle e^{-\imath \omega_{0} t}\right] \\
& =\frac{1}{4 \omega_{0}} \operatorname{cosech} \frac{\beta \omega_{0}}{2}\left[e^{-\imath \omega_{0} t+\beta \omega_{0} / 2}+e^{\imath \omega_{0} t-\beta \omega_{0} / 2}\right] .
\end{aligned}
$$

$\mathrm{Use}^{3} e^{y(s+1 / s) / 2}=\sum_{n=-\infty}^{+\infty} s^{n} \mathrm{I}_{n}(y)$ with $y \doteq \frac{q^{2}}{2 \omega_{0}} \operatorname{cosech} \frac{\beta \omega_{0}}{2}, s \doteq e^{-\imath \omega_{0} t+\beta \omega_{0} / 2}$.

$$
\begin{gathered}
\tilde{S}_{A A}(q, t)=e^{-2 W} \sum_{n=-\infty}^{+\infty} \mathrm{I}_{n}\left(\frac{q^{2}}{2 \omega_{0}} \operatorname{cosech} \frac{\beta \omega_{0}}{2}\right) \exp \left(\frac{1}{2} \beta n \omega_{0}-\imath n \omega_{0} t\right) . \\
S_{A A}(q, \omega)=e^{\beta \omega / 2-2 W} \sum_{n=-\infty}^{+\infty} \mathrm{I}_{n}\left(\frac{q^{2}}{2 \omega_{0}} \operatorname{cosech} \frac{\beta \omega_{0}}{2}\right) \delta\left(\omega-n \omega_{0}\right)
\end{gathered}
$$

\footnotetext{
${ }^{1}$ We consider component of displacement parallel to $\mathbf{q} \doteq \mathbf{k}_{\mathrm{f}}-\mathbf{k}_{\mathrm{i}}$ only.

${ }^{2}$ Use also $\left\langle e^{A}\right\rangle=e^{\left\langle A^{2}\right\rangle / 2}$ for linear combinations of boson operators.

${ }^{3} \mathrm{I}_{n}(y)$ are modified Bessel functions of the first kind. Note that $\mathrm{I}_{-n}(y)=\mathrm{I}_{n}(y)$.
} 


\section{Scattering from Harmonic Crystal [nnn95]}

Atoms of mass $M$ are harmonically coupled via a bilinear form in displacement coordinates. Spatial Fourier transform produces normal modes: noninteracting collective excitations (phonons) representing oscillating patterns of specific wave vectors $\mathbf{k}$ and excitation energies determined by a characteristic dispersion relation $\epsilon(\mathbf{k})$.

$$
\mathcal{H}=\sum_{l} \frac{p_{l}^{2}}{2 M}+\frac{1}{2} \sum_{l, l^{\prime}} \mathbf{u}_{l} \cdot \mathbf{D}_{l l^{\prime}} \cdot \mathbf{u}_{l^{\prime}}=\sum_{\mathbf{k}} \epsilon(\mathbf{k}) a_{\mathbf{k}}^{\dagger} a_{\mathbf{k}}
$$

Correlation function: ${ }^{1}$

$$
\begin{aligned}
\tilde{S}(\mathbf{q}, t) & =\left\langle e^{-\imath \mathbf{q} \cdot \mathbf{u}_{l}} e^{\imath \mathbf{q} \cdot \mathbf{u}_{l^{\prime}}(-t)}\right\rangle \\
& =\exp \left(-\frac{1}{2}\left\langle\left[\mathbf{q} \cdot \mathbf{u}_{l}\right]^{2}\right\rangle-\frac{1}{2}\left\langle\left[\mathbf{q} \cdot \mathbf{u}_{l^{\prime}}(-t)\right]^{2}\right\rangle+\left\langle\left[\mathbf{q} \cdot \mathbf{u}_{l}\right]\left[\mathbf{q} \cdot \mathbf{u}_{l^{\prime}}(-t)\right]\right\rangle\right)
\end{aligned}
$$

Debye-Waller factor from $\frac{1}{2}\left\langle\left[\mathbf{q} \cdot \mathbf{u}_{l}\right]^{2}\right\rangle=\frac{1}{2}\left\langle\left[\mathbf{q} \cdot \mathbf{u}_{l^{\prime}}(-t)\right]^{2}\right\rangle=W$.

Expansion into $m$-phonon processes:

$$
\exp \left(\left\langle\left[\mathbf{q} \cdot \mathbf{u}_{l}\right]\left[\mathbf{q} \cdot \mathbf{u}_{l^{\prime}}(-t)\right]\right\rangle\right)=\sum_{m=0}^{\infty} \frac{1}{m !}\left(\left\langle\left[\mathbf{q} \cdot \mathbf{u}_{l}\right]\left[\mathbf{q} \cdot \mathbf{u}_{l^{\prime}}(-t)\right]\right\rangle\right)^{m}
$$

Dynamic structure factor:

$$
S(\mathbf{q}, \omega)=e^{-2 W} \frac{1}{N} \sum_{l l^{\prime}} e^{\imath \mathbf{q} \cdot\left(\mathbf{R}_{l}-\mathbf{R}_{l^{\prime}}\right)} \int_{-\infty}^{+\infty} d t e^{\imath \omega t} \exp \left(\left\langle\left[\mathbf{q} \cdot \mathbf{u}_{l}\right]\left[\mathbf{q} \cdot \mathbf{u}_{l^{\prime}}(-t)\right]\right\rangle\right) .
$$

$m=0:$ Bragg scattering

$$
S(\mathbf{q}, \omega)_{0} \propto e^{-2 W} \delta(\omega) \sum_{\mathbf{G}} \delta_{\mathbf{q}, \mathbf{G}} ; \quad \mathbf{G}: \text { reciprocal lattice vector. }
$$

$m=1:$ 1-phonon contributions ${ }^{2}$

$$
S(\mathbf{q}, \omega)_{1} \propto e^{-2 W} \frac{[\mathbf{q} \cdot \mathbf{e}(\mathbf{k})]^{2}}{2 M \epsilon(\mathbf{k})}(\underbrace{\left[1+n_{\mathrm{B}}(\mathbf{q})\right] \delta(\omega-\epsilon(\mathbf{q}))}_{\text {phonon emission }}+\underbrace{n_{\mathrm{B}}(\mathbf{q}) \delta(\omega+\epsilon(\mathbf{q}))}_{\text {phonon absorption }}) .
$$

Harmonicity leaves phonon peaks sharp. Thermal fluctuations only affect intensity via Debye-Waller factor.

\footnotetext{
${ }^{1}$ Use $\left\langle e^{A} e^{B}\right\rangle=e^{\left\langle A^{2}+2 A B+B^{2}\right\rangle / 2}$ for operators $A, B$ that are linear in $\mathbf{u}_{l}, \mathbf{p}_{l}$.

${ }^{2}$ Calculate $\left\langle\left[\mathbf{q} \cdot \mathbf{u}_{\mathbf{0}}\right]\left[\mathbf{q} \cdot \mathbf{u}_{\mathbf{R}}(-t)\right]\right\rangle \quad$ with $\quad \mathbf{u}_{\mathbf{R}} \propto \sum_{\mathbf{k}}(2 M \epsilon(\mathbf{k}))^{-1 / 2}\left(a_{\mathbf{k}}+a_{\mathbf{k}}^{\dagger}\right) e^{\imath \mathbf{k} \cdot \mathbf{R}} \mathbf{e}(\mathbf{k})$ and $a_{\mathbf{k}}(t)=a_{\mathbf{k}} e^{-\imath \epsilon(\mathbf{k}) t}$.
} 


\section{Magnetic Resonance or Scattering [nnngr]}

Magnetic probe: $\mathcal{H}_{1}(t)=-\mathbf{M} \cdot \mathbf{h}(t) . \quad$ We set $\hbar=1$ throughout.

Linear response: $\left\langle M_{\mu}(\mathbf{r}, t)\right\rangle-\left\langle M_{\mu}\right\rangle_{\mathrm{eq}}=\sum_{\nu} \int d^{3} r^{\prime} \int d t \tilde{\chi}_{\mu \nu}\left(\mathbf{r}-\mathbf{r}^{\prime}, t-t^{\prime}\right) h_{\nu}\left(\mathbf{r}^{\prime}, t^{\prime}\right)$.

Response function: $\left.\tilde{\chi}_{\mu \nu}(\mathbf{r}, t)=\imath \theta(t)\left\langle\left[M_{\mu}(\mathbf{r}, t), M_{\nu}(\mathbf{0}, 0)\right]\right\rangle=\imath \theta(t)\left[S_{\mathbf{1}+\mathbf{r}}^{\mu}(t), S_{\mathbf{l}}^{\nu}\right]\right\rangle$.

Generalized susceptibility: $\quad \chi_{\mu \nu}(\mathbf{q}, \omega)=\sum_{\mathbf{r}} e^{\imath \mathbf{q} \cdot \mathbf{r}} \int_{-\infty}^{+\infty} d t e^{\imath \omega t} \tilde{\chi}_{\mu \nu}(\mathbf{r}, t)$.

Correlation function: $\tilde{S}_{\mu \nu}(\mathbf{r}, t)=\left\langle S_{\mathbf{1}+\mathbf{r}}^{\mu}(t) S_{\mathbf{l}}^{\nu}\right\rangle$.

Dynamic structure factor: $S_{\mu \nu}(\mathbf{q}, \omega)=\sum_{\mathbf{r}} e^{\imath \mathbf{q} \cdot \mathbf{r}} \int_{-\infty}^{+\infty} d t e^{\imath \omega t} \tilde{S}_{\mu \nu}(\mathbf{r}, t)$.

Relation from $[\operatorname{nn} 39]: \quad S_{\mu \nu}(\mathbf{q}, \omega)=\frac{2 \chi_{\mu \nu}^{\prime \prime}(\mathbf{q}, \omega)}{1-e^{-\beta \omega}}$.

Experimental techniques:

- Ferromagnetic resonance, EPR.

- Long wavelengths (long compared to lattice spacing) probed.

- Relevant quantitity: $\chi_{\mu \nu}^{\prime \prime}(\mathbf{q} \simeq 0, \omega)$.

- Inelastic neutron scattering.

- Interaction with magnetic dipole moment of neutron.

- Momentum transfer $\mathbf{q}$ and energy transfer $\omega$ of neutrons well matched with energy-momentum relations $\epsilon(\mathbf{q})$ of typical collective magnetic excitations.

- Scattering cross section: $\frac{d^{2} \sigma}{d \omega d \Omega} \propto S_{\mu \nu}(\mathbf{q}, \omega)$.

- Nuclear magnetic resonance, NMR.

- Localized probe (nuclear magnetic moment) interacts with electronic magnetism in immediate vicinity.

- Spin-lattice relaxation rate: $\frac{1}{T_{1}} \propto \sum_{\mathbf{q}} S_{\mu \nu}\left(\mathbf{q}, \omega_{\mathrm{N}}\right)$.

- Nuclear Larmor frequency $\omega_{\mathrm{N}}$ is very small compared to typical electronic magnetic excitations. 